\title{
DENSIDADE BÁSICA E ESTIMATIVA DE MASSA SECA E DE LIGNINA NA MADEIRA EM ESPÉCIES DE Eucalyptus
}

\author{
Basic density and dry mass and lignin mass estimate in Eucalyptus wood species
}

\author{
Paulo Fernando Trugilho ${ }^{1}$
}

\begin{abstract}
RESUMO
Objetivou-se, no presente trabalho, usar três diferentes formas para estimar a densidade básica da madeira, visando a obtenção da estimativa de massa seca e a massa de lignina na madeira em nove espécies de Eucalyptus, em diferentes idades. Foram usadas três formas de estimativa da densidade básica da madeira, ou seja, em um único ponto no tronco (1,3 $\mathrm{m}$ de altura do solo), a média ponderada pelo volume e a média aritmética. Essas estimativas foram usadas para a determinação da massa seca e de lignina na madeira. Os resultados indicam que o Eucalyptus grandis W.Hill. (EG), aos quatro anos, e o híbrido (HB), nas demais idades, foram as espécies que se destacaram no incremento volumétrico. $\mathrm{O}$ efeito da forma de estimativa somente foi significativo para a densidade básica nas idades de cinco, seis e sete anos. O HB foi à única espécie que apresentou diferença entre as formas de estimativa de densidade básica da madeira. O EG e o HB foram as espécies que se destacaram na produção de massa seca e de lignina, apesar de não serem as madeiras de maior densidade básica e maior teor de lignina. $\mathrm{O}$ incremento volumétrico desempenhou importante papel nas estimativas de massa seca e de lignina na madeira e, portanto, deve ser considerado em todas as utilizações da madeira que depender dessas estimativas.
\end{abstract}

Termos para indexação: Densidade básica, madeira, Eucalyptus.

\begin{abstract}
The objective of the research was to use three different forms to calcuate the basic wood density of trees to obtain the estimate of dry mass and lignin mass of nine Eucalyptus wood species, at different ages. Three different forms were used to estimate the basic density of wood, at only one point on the trunk (1,3 $\mathrm{m}$ of height from the ground), the average weighted estimate by volume, and the arithmetical average. These estimates were used for the determination in the estimates of dry mass and lignin mass in the wood. The results showed the Eucalyptus grandis W. Hill., at four years of age, and the hybrid, at other ages were the species that highlighted in volumetric increment. The effects of species, age, and interaction species $\mathrm{x}$ age were significant for all the evaluated features. The effect of the estimate form was only significant for the basic density at ages five, six, and seven years. The hybrid was the only species that presented a difference in the forms of estimating basic wood density. Eucalyptus grandis and the hybrid were the species that highlighted in the production of dry mass and lignin, in spite of not being wood with the greater basic density and lignin content. The volumetric increment played an important role in the estimates of dry mass and lignin in the wood. So, it must be considered in all applications of wood that depend on these estimates.
\end{abstract}

Index terms: Basic density, Wood, Eucalyptus.

(Recebido em 17 de outubro de 2006 e aprovado em 17 de setembro de 2007)

\section{INTRODUÇÃO}

Segundo Brito et al. (1984) o Eucalyptus é um importante gênero fornecedor de matéria-prima para diversas finalidades industriais. Várias das espécies desse gênero se adaptaram perfeitamente às nossas condições edafoclimáticas e passaram a ser importantes matériasprimas para a produção de lenha, carvão vegetal, celulose, entre outros. Segundo o Ministério de Minas e Energia (2005) a lenha somada ao carvão vegetal participa com $11,5 \%$ do consumo por fonte na matriz energética nacional. Grande parte desse consumo advém da madeira do gênero Eucalyptus, que é amplamente plantado no Brasil.

No estado de Minas Gerais, o Eucalyptus é o principal fornecedor de carvão vegetal para o abastecimento do setor siderúrgico. De acordo com a Associação Mineira de Silvicultura (2006), Minas Gerais consumiu $66,1 \%$ de todo o carvão vegetal produzido no Brasil em 2005, demonstrando a importância desse produto para o Estado. O carvão vegetal é um produto que sofre influência direta da madeira de origem. Assim, existem potencialidades diferentes de acordo com a espécie utilizada. Algumas das características intrínsecas da madeira que afetam a produção de carvão vegetal são a densidade básica e o teor de lignina na madeira. Todavia, o incremento volumétrico da árvore pode influenciar significativamente tal produção, uma vez que afeta diretamente a quantidade de massa seca do indivíduo. Segundo Botrel (2006) os ganhos esperados, na seleção de clones, são de 25,55,

${ }^{1}$ Engenheiro Florestal, Doutor, Professor - Departamento de Ciências Florestais/DCF - Universidade Federal de Lavras/UFLA - Cx. P. 3037 - $37200-000$ Lavras, MG - trugilho@ufla.br 
6,98, 27,58, 9,36 e 33,46\%, respectivamente, para o volume individual, densidade básica, massa seca estimada, teor de lignina e massa de lignina estimada na madeira. Demonstrando a importância que as estimativas de massa seca e de lignina, apresentam sobre a seleção de materiais genéticos destinados aos usos energéticos da biomassa.

Dessa forma, as estimativas de biomassa seca e de lignina passam a ser fatores de fundamental importância quando a madeira é destinada para a produção de energia, tanto na forma de lenha como de carvão vegetal, e também para a fabricação de celulose e papel e chapas de fibras. Segundo Oliveira et al. (1991) a quantificação de massa de madeira é preferível para esses usos, mais do que a utilização dos parâmetros dendrométricos, como, por exemplo, o volume de madeira.

Atualmente, várias empresas do setor florestal utilizam a informação da estimativa de massa de madeira por área para fazer previsão da quantidade de produtos a ser gerado por material genético e por área. Empresas como as de produção de celulose já conseguem estimar a produção de celulose por clone, por hectare e por ano. Essa nova característica está sendo chamada de imacel, ou seja, incremento médio de celulose. As empresas que produzem carvão vegetal também estão procurando fazer algo semelhante, ou seja, estimar o imacarv, incremento médio em carvão vegetal. Para que isso possa se tornar uma realidade é preciso estimar com precisão a densidade básica do material genético e determinar o seu incremento volumétrico, para poder estimar a massa seca produzida por área.

Para se estimar a massa seca de madeira pode-se optar por diversos métodos ou procedimentos. Uma alternativa é usar modelos de regressão ajustados para dada localidade, com os quais se determine a relação entre a massa estimada, o diâmetro e a altura das árvores, em amostra representativa da área. Esse procedimento usa o volume do fuste comercial das árvores e a densidade básica média dos indivíduos. O método depende do tamanho da amostra e da intensidade da amostragem da densidade básica ao longo do fuste das árvores. Sabe-se que a densidade básica da madeira varia tanto no sentido transversal como no longitudinal ao fuste das árvores. De acordo com Downes et al. (1997) diferentes formas de amostragens longitudinais no tronco das árvores podem levar a diferentes estimativas da densidade básica da madeira. Conseqüentemente, tanto o tipo de amostragem quanto a forma e o método de obtenção da estimativa da densidade básica podem promover diferenças nas estimativas de massa seca e nas estimativas de produtos por área.
Objetivou-se, no presente trabalho usar três diferentes formas para estimar a densidade básica da madeira, visando a obtenção da estimativa de massa seca e a massa de lignina na madeira em nove espécies de Eucalyptus, em diferentes idades.

\section{MATERIAL E MÉTODOS}

\section{Material Experimental}

Foram utilizadas, neste estudo, árvores-amostras de nove espécies de Eucalyptus, de diferentes idades, procedentes de povoamentos implantados pela COPENER, em Alagoinhas-BA, e pela PAINS FLORESTAL, em Três Marias-MG, conforme descrito na Tabela 1 . As árvores foram plantadas em espaçamento comercial de 3 x $2 \mathrm{~m}$.

As árvores-amostras selecionadas nos povoamentos foram aquelas cujos diâmetro à $1,30 \mathrm{~m}$ de altura do solo (DAP) estava compreendido entre o DAP médio estimado para a população o seu desvio padrão $(\overline{\mathrm{X}} \pm \mathrm{DP})$. Foram amostradas cinco árvores-amostras por espécie e por idade, resultando em um total de 85 árvores. Tomou-se o cuidado de escolher somente árvores em bom estado fitossanitário e evitou-se aquelas plantadas nas bordas dos talhões.

De cada árvore amostra foram retirados toretes de $30 \mathrm{~cm}$ de comprimento a 0 (base), 25, 50, 75 e $100 \%$ da altura comercial do tronco (VITAL, 1984), que foi considerada até um diâmetro mínimo de $5 \mathrm{~cm}$. Nessas posições, foram medidos os diâmetros com e sem casca para a determinação do volume individual de madeira por espécie e idade. Um torete extra foi retirado a 1,3 m de altura do solo (DAP). Na porção mediana de cada torete, foi retirado um disco de $2,5 \mathrm{~cm}$ de espessura, $\mathrm{o}$ qual foi subdividido em quatro partes, em forma de cunha, passando pela medula. Uma das partes foi destinada à determinação da densidade básica e outra foi destinada à determinação do teor de lignina na madeira. As duas partes restantes foram armazenadas em caso de necessidade posterior. As partes selecionadas, por altura do tronco, foram convenientemente identificadas e utilizadas para representar a árvore inteira. Os cinco pontos amostrados $(0,25,50,75$ e $100 \%$ da altura comercial) foram utilizados na determinação da densidade básica e teor de lignina. O torete retirado na altura do DAP foi utilizado para determinar somente a densidade básica da madeira. Todas as árvores-amostra foram cubadas rigorosamente para a determinação do seu volume individual. Na Figura 1 apresenta-se o esquema da amostragem usada nas árvores selecionadas. 
Tabela 1 - Relação das espécies de Eucalyptus utilizadas.

\begin{tabular}{|c|c|c|c|c|c|}
\hline Código & Espécie & $\begin{array}{l}\text { Idade } \\
\text { (Anos) }\end{array}$ & Local de Coleta & Procedência & Subgênero \\
\hline EG & E. grandis & 4 & COPENER & Paluma & Symphyomyrtus \\
\hline EU & E. urophylla & 4 & $\begin{array}{c}\text { PAINS } \\
\text { FLORESTAL }\end{array}$ & Itamarandiba** & Symphyomyrtus \\
\hline $\mathrm{ECl}$ & E. cloeziana & 4 & $\begin{array}{c}\text { PAINS } \\
\text { FLORESTAL }\end{array}$ & Capelinha $* * *$ & Idiogenes \\
\hline ER & E. resinifera & 4 & $\begin{array}{c}\text { PAINS } \\
\text { FLORESTAL }\end{array}$ & Lewis Track QD & Symphyomyrtus \\
\hline $\mathrm{ECm}$ & E. camaldulensis & 4 & $\begin{array}{c}\text { PAINS } \\
\text { FLORESTAL }\end{array}$ & Bom Despacho**** & Symphyomyrtus \\
\hline EM & E. maculata & 7 & COPENER & Gympie & Corymbia \\
\hline EP & E. pellita & 7 & COPENER & Morada Nova* & Symphyomyrtus \\
\hline ET & E. tereticornis & 7 & COPENER & Mareeba & Symphyomyrtus \\
\hline EU & E. urophylla & 7 & COPENER & Lençóis Paulista & Symphyomyrtus \\
\hline HB & Híbrido & 7 & COPENER & Aracruz & Symphyomyrtus \\
\hline $\mathrm{ECl}$ & E. cloeziana & 7 & COPENER & Mtao Forest & Idiogenes \\
\hline \multirow{3}{*}{ EP } & E. pellita & 5 & COPENER & Helenvale & Symphyomyrtus \\
\hline & E. pellita & 6 & COPENER & Morada Nova* & Symphyomyrtus \\
\hline & E. pellita & 7 & COPENER & Morada Nova* & Symphyomyrtus \\
\hline \multirow{3}{*}{ EU } & E. urophylla & 5 & COPENER & Guarujá & Symphyomyrtus \\
\hline & E. urophylla & 6 & COPENER & Avaré & Symphyomyrtus \\
\hline & E. urophylla & 7 & COPENER & Lençóis Paulista & Symphyomyrtus \\
\hline \multirow{3}{*}{ HB } & HÍBRIDO & 5 & COPENER & Aracruz & Symphyomyrtus \\
\hline & HÍBRIDO & 6 & COPENER & Aracruz & Symphyomyrtus \\
\hline & HÍBRIDO & 7 & COPENER & Aracruz & Symphyomyrtus \\
\hline
\end{tabular}

* Origem Helenvale, ** Origem Timor, *** Origem Gympie, **** Origem Petford.

Híbrido = Cruzamento entre o Eucalyptus grandis com o Eucalyptus urophylla .

A densidade básica média ponderada pelo volume foi estimada utilizando-se a equação 1 e a densidade básica média aritmética da árvore foi obtida pela equação 2 .

$$
\mathrm{DBmP}=\frac{\left(\mathrm{DBm}_{(0-25 \%)} \times \mathrm{V}_{(0-25 \%)}\right)+\cdots+\left(\mathrm{DBm}_{(75-100 \%)} \times \mathrm{V}_{(75-100 \%)}\right)}{\mathrm{V}_{(0-25 \%)}+\mathrm{V}_{(25-50 \%)}+\mathrm{V}_{(50-75 \%)}+\mathrm{V}_{(75-100 \%)}}
$$

em que, DBmP é a densidade básica média ponderada da árvore, $\mathrm{g} / \mathrm{cm}^{3}$; DBm é a densidade básica média entre as posições da base (0\%) e $25 \%, 25 \%$ e $50 \%, 50 \%$ e $75 \%$ e
$75 \%$ e $100 \%$ da altura comercial, $\mathrm{g} / \mathrm{cm}^{3}$; V é o volume das seções entre as posições da base $(0 \%)$ e $25 \%, 25 \%$ e $50 \%$, $50 \%$ e $75 \%$ e $75 \%$ e $100 \%$ da altura comercial, $\mathrm{m}^{3}$.

$$
\mathrm{DBmA}=\frac{\mathrm{DB}_{0 \%}+\mathrm{DB}_{25 \%}+\mathrm{DB}_{50 \%}+\mathrm{DB}_{75 \%}+\mathrm{DB}_{100 \%}}{5}
$$

on de, DBmA é a densidade básica média aritmética da árvore, $\mathrm{g} / \mathrm{cm}^{3} ; \mathrm{DBm}$ é a densidade básica média por ponto de amostragem, $\mathrm{g} / \mathrm{cm}^{3}$. 


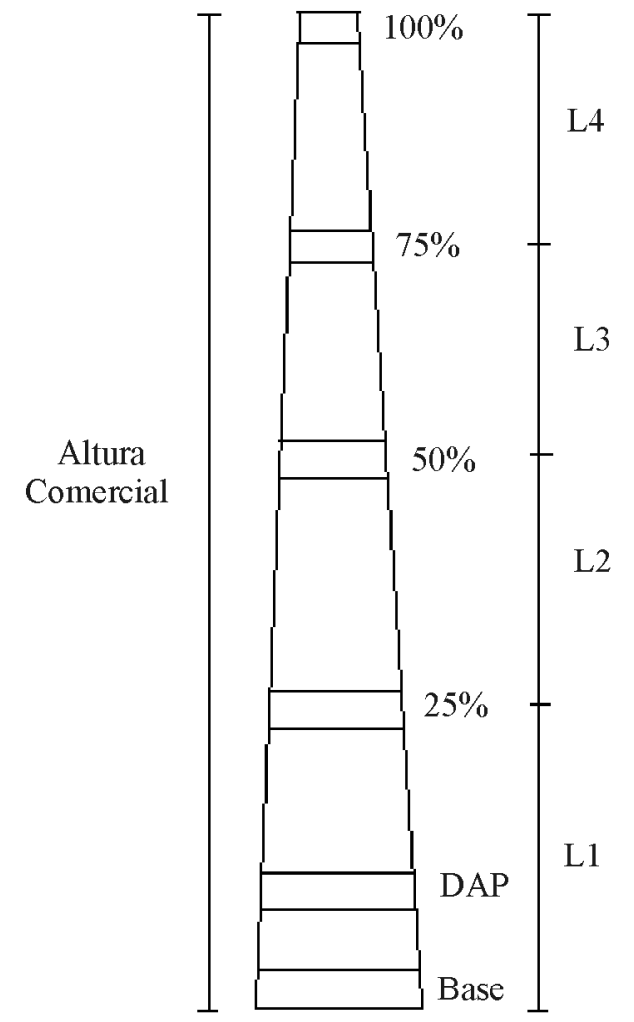

Figura 1 - Esquema da forma de amostragem usada nas árvores.

\section{Características Avaliadas na Madeira}

As características avaliadas na madeira foram a densidade básica e teor de lignina Klason. A densidade básica foi determinada de acordo com o método de imersão em água, descrito por Vital (1984), em cada ponto amostrado no tronco das árvores. Foram considerados os valores de densidade básica média estimada por diferentes formas: pela média ponderada pelo volume; pela média aritmética considerando-se os pontos de amostragem longitudinal no tronco (base, 25, 50, 75 e 100\% da altura comercial); e pelo valor obtido na posição do DAP.

O teor de lignina (Klason) foi determinado de acordo com o procedimento descrito por Gomide \& Demuner (1986). O teor de lignina solúvel em ácido sulfúrico foi determinado por meio da espectrofotometria, tendo sido utilizada a equação descrita por Goldschimid (1971) (equação 3). A lignina total foi tomada como sendo a soma das ligninas solúvel e insolúvel. O teor de lignina foi determinado por meio de uma amostra composta por material proveniente dos cincos pontos eqüidistantes considerados no tronco de cada árvore-amostra.

$$
\operatorname{TLS}(\%)=\frac{\left(4,53 \times \mathrm{A}_{215}\right)-\mathrm{A}_{280}}{300 \times \mathrm{P}} \times 100
$$

em que, $\mathrm{A}_{215}$ é a Absorbância do filtrado em $215 \mathrm{~nm} ; \mathrm{A}_{280}$ é a Absorbância do filtrado em $280 \mathrm{~nm}$; P é o peso da amostra de madeira usada, em gramas.

\section{Estimativa de Massa Seca e de Lignina}

As estimativas de massa seca e de lignina foram obtidas a partir das três diferentes formas de estimativa de densidade básica da madeira aplicados nesse trabalho.

\section{Delineamento Experimental Adotado}

$\mathrm{Na}$ avaliação experimental adotou-se o delineamento inteiramente casualizado com cinco repetições (5 árvoresamostras). Dentre as variáveis dendrométricas observadas nas árvores (DAP com e sem casca; ht e hc; V com e sem casca), optou-se por avaliar somente os volumes, com e sem casca. Para os volumes, foi avaliado o efeito da espécie para a idade de quatro e sete anos, além dos efeitos de espécie e idade para cinco, seis e sete anos. Para a densidade básica, estimativa de massa seca e de lignina 
foi usado o delineamento inteiramente casualizado disposto em esquema fatorial com dois fatores, espécies e método, para a idade de quatro e sete anos. Para os materiais com cinco, seis e sete anos de idade foram avaliados três fatores, ou seja, espécie, idade e método. Para o teor de lignina foi avaliado somente o efeito da espécie para a idade de quatro e sete anos, além dos efeitos de espécie e idade para cinco, seis e sete anos de idade. Foram avaliados também todos os efeitos das interações. Os modelos estatísticos associados aos experimentos estão representados nas equações $4,5,6$ e 7 .

$$
Y_{i j}=\mu+E_{i}+e_{i j}
$$

onde, $\mathrm{Y}_{\mathrm{ij}}$ é a i-ésima observação da j-ésima repetição para os volumes, com e sem casca, e o teor de lignina da madeira aos 4 e 7 anos de idade, $m$ é a média geral, $E_{i}$ é o efeito da iésima espécie.

$$
\mathrm{Y}_{\mathrm{ijk}}=\mu+\mathrm{E}_{\mathrm{i}}+\mathrm{I}_{\mathrm{j}}+(\mathrm{EI})_{\mathrm{ij}}+\mathrm{e}_{\mathrm{ijk}}
$$

em que, $\mathrm{Y}_{\mathrm{ijk}}$ é a i-ésima observação da j-ésima idade na késima repetição para os volumes, com e sem casca, e o teor de lignina da madeira aos 5, 6 e 7 anos de idade, I é o efeito da j-ésima idade, $(E I)_{i j}$ é o efeito da interação espécie x idade.

$$
\mathrm{Y}_{\mathrm{ilk}}=\mu+\mathrm{E}_{\mathrm{i}}+\mathrm{F}_{1}+(\mathrm{EF})_{\mathrm{il}}+\mathrm{e}_{\mathrm{ilk}}
$$

onde, $\mathrm{Y}_{\text {ilk }}$ é a i-ésima observação da 1-ésima forma de estimativa na k-ésima repetição para a densidade básica e estimativas de massa seca e de lignina na madeira, aos $4 \mathrm{e}$ 7 anos de idade, $\mathrm{F}_{1}$ é o efeito da l-ésima forma de estimativa, $(\mathrm{EF})_{\mathrm{il}}$ é o efeito da interação espécie x forma.

$\mathrm{Y}_{\mathrm{ijjk}}=\mu+\mathrm{E}_{\mathrm{i}}+\mathrm{I}_{\mathrm{j}}+\mathrm{F}_{1}+(\mathrm{EI})_{\mathrm{ij}}+(\mathrm{EF})_{\mathrm{il}}+(\mathrm{IF})_{\mathrm{j} 1}+(\mathrm{EIF})_{\mathrm{ij} 1}+\mathrm{e}_{\mathrm{ijjk}}$

em que, $Y_{i j k}$ é a i-ésima observação da j-ésima espécie na lésima forma de estimativa na k-ésima repetição para a densidade básica e as estimativas de massas aos 5, 6 e 7 anos de idade, $(\mathrm{IF})_{\mathrm{j} 1}$ é o efeito da interação idade $\mathrm{x}$ forma de estimativa, $(\mathrm{EIF})_{\mathrm{ijl}}$ é a interação tripla. Os erros experimentais são representados por $e_{i j}, e_{i j k}, e_{i l k} e_{i j l k}$.

\section{RESULTADOS E DISCUSSÃO}

\section{Volumes com e sem casca}

Na Tabela 2 apresenta-se o resumo da análise de variância para a característica volume, com e sem casca, das espécies e idades avaliadas. Observa-se que o efeito de idade foi significativo para quatro, sete e cinco, seis e sete anos de idade. O efeito da idade e da interação espécie $\mathrm{x}$ idade foram também significativos, o que era esperado. Interação significativa indica que existe dependência entre os fatores espécie e idade. Dessa forma, realizou-se o desdobramento da interação e avaliação do efeito de idade, dentro de espécies.

Os resultados médios e o teste de comparação múltipla para o volume, com e sem casca, das espécies de Eucalyptus estão apresentados na Tabela 3. Observa-se, pela Tabela 3, que existe uma tendência geral de aumento nos diâmetros e alturas e, conseqüentemente, nos volumes com o aumento da idade do material. Analisando a idade de quatro anos, verifica-se que o Eucalyptus grandis W. Hill. (EG) apresentou o maior volume, destacando-se em relação aos demais. Esse resultado certamente está associado à localidade e às diferentes condições edafoclimáticas dos municípios de Alagoinhas-BA e Três Marias-MG. Para sete anos de idade o destaque foi o híbrido (HB), o qual apresentou um volume, sem casca, 390\% superior ao do Eucalyptus tereticornis Sm. (ET), espécie de menor volume médio.

Considerando os materiais nas três diferentes idades observa-se que o Eucalyptus pellita F. Muell. (EP) apresentou aumento de $31,17 \%$, do quinto para o sexto ano, e um decréscimo de 7,39\%, do sexto para o sétimo ano de idade, no volume, sem casca. Essa espécie apresentou um ligeiro aumento no volume, com casca, do sexto para o sétimo ano de idade (1,38\%). O Eucalyptus urophylla S.T. Blake (EU) apresentou queda de $10,43 \%$, do quinto para o sexto ano, e um aumento de $135,87 \%$, do sexto para o sétimo ano de idade, no volume, sem casca. O híbrido apresentou aumento de $38,17 \%$ e $44,62 \%$ no volume, sem casca, do quinto para o sexto e do sexto para o sétimo ano de idade, respectivamente. O EP não apresentou diferença estatística para os volumes, tanto com como sem casca, entre as idades avaliadas, indicando uma estabilização do volume a partir do sexto ano. O EU apresentou diferença estatística significativa para os volumes, com e sem casca, do sexto para o sétimo ano de idade. O HB apresentou diferença estatística significativa entre os volumes com e sem casca, entre todas as idades avaliadas. Esse fato indica que tanto o EU como HB ainda estão em franco crescimento aos sete anos de idade. 
Tabela 2 - Resumo da análise de variância para o volume, com e sem casca, das espécies aos quatro, sete e cinco, seis e sete anos de idade

\begin{tabular}{|c|c|c|c|c|}
\hline \multirow[b]{3}{*}{$\mathrm{FV}$} & \multicolumn{4}{|c|}{ Quatro Anos } \\
\hline & \multicolumn{2}{|c|}{ Volume $\mathrm{CC}\left(\mathrm{m}^{3}\right)$} & \multicolumn{2}{|c|}{ Volume $\mathrm{SC}\left(\mathrm{m}^{3}\right)$} \\
\hline & GL & QM & GL & QM \\
\hline Espécie & 4 & $0,022376 * *$ & 4 & $0,017581 * *$ \\
\hline Resíduo & 20 & 0,000621 & 20 & 0,000485 \\
\hline \multirow[t]{2}{*}{$\mathrm{CV}(\%)$} & \multicolumn{2}{|c|}{28,35} & \multicolumn{2}{|c|}{31,50} \\
\hline & \multicolumn{4}{|c|}{ Sete Anos } \\
\hline $\mathrm{FV}$ & GL & QM & GL & QM \\
\hline Espécie & 5 & $0,089249 * *$ & 5 & $0,071585 * *$ \\
\hline Resíduo & 24 & 0,002835 & 24 & 0,002009 \\
\hline \multirow[t]{2}{*}{$\mathrm{CV}(\%)$} & \multicolumn{2}{|c|}{23,92} & \multicolumn{2}{|c|}{24,67} \\
\hline & \multicolumn{4}{|c|}{ Cinco, Seis e Sete Anos } \\
\hline $\mathrm{FV}$ & GL & QM & GL & QM \\
\hline Espécie (E) & 2 & $0,209388 * *$ & 2 & 0,183533 ** \\
\hline Idade (I) & 2 & $0,064231 * *$ & 2 & $0,041907 * *$ \\
\hline Ex I & 4 & $0,015468 * *$ & 4 & $0,013291 * *$ \\
\hline Resíduo & 36 & 0,002731 & 36 & 0,001877 \\
\hline $\mathrm{CV}(\%)$ & \multicolumn{2}{|c|}{23,53} & \multicolumn{2}{|c|}{23,87} \\
\hline
\end{tabular}

$\mathrm{FV}=$ fonte de variação; $\mathrm{GL}=$ grau de liberdade, $\mathrm{QM}=$ quadrado médio, $\mathrm{CC}$ e $\mathrm{SC}=$ com e sem casca, $* *=$ significativo a $1 \%$ de probabilidade.

\section{Características avaliadas na madeira}

As Tabelas 4 e 5 apresentam os resumos das análises de variância para as características avaliadas na madeira. Pela Tabela 4, observa-se que, para quatro e sete anos de idade, somente o efeito de espécie foi significativo para a densidade básica, estimativa de massa seca e de lignina na madeira. A interação espécie x forma de estimativa foi não significativa, indicando a independência entre os dois fatores. O efeito da forma de estimativa de determinação foi não-significativo, indicando a não-existência de diferença estatística entre as formas de estimativa usadas, tanto para a densidade básica como as massas.

Pela Tabela 4, observa-se que, para as idades de cinco, seis e sete anos de idade, os efeitos de espécie, idade e interação espécie $\mathrm{x}$ idade foram significativos para as três características avaliadas. $\mathrm{O}$ efeito de forma de estimativa foi significativo somente para a densidade básica da madeira. $\mathrm{O}$ efeito significativo da interação espécie $\mathrm{x}$ idade era esperado, pois essa característica sofre influência direta da idade. Vital et al. (1984) observaram aumento linear da densidade básica do tronco de árvores de Eucalyptus grandis de um ao sétimo ano de idade. Resultado idêntico foi encontrado por Trugilho et al. (1996) para o Eucalyptus saligna Sm.de 12 a 48 meses de idade. Interação significativa indica a existência de dependência entre os fatores espécie e idade, assim optou-se por realizar o desdobramento da interação e avaliação do efeito de idade dentro de espécies.

Pela Tabela 5, observa-se que o efeito de espécie foi significativo para o teor de lignina em todas as idades consideradas. Para as três idades diferentes, o efeito da interação espécie $\mathrm{x}$ idade foi significativo, indicando a existência de dependência entre os dois fatores. Trugilho et al. (1996) encontraram relação de decréscimo no teor de lignina com o aumento da idade, em Eucalyptus saligna. Os autores indicaram que a redução é acentuada de 12 para 36 meses de idade, tendendo a uma estabilização de 36 para 48 meses de idade.

Na Tabela 6, apresentam-se os resultados médios da densidade básica e massa seca estimada na madeira das espécies avaliadas e o teste de comparação múltipla. Pode-se observar que as estimativas de massa seca obtidas pela densidade básica média ponderada pelo volume e pela densidade básica media aritmética foram bem próximas, em todas as espécies e idades consideradas. A estimativa de massa seca obtida com a densidade básica a 1,3 $\mathrm{m}$ de altura do solo (DAP), na maioria dos casos, apresentou valor ligeiramente inferior em relação às outras duas formas de 
Tabela 3 - Valores médios das características de crescimento.

\begin{tabular}{|c|c|c|c|c|c|c|c|}
\hline \multirow[b]{2}{*}{ Espécie } & \multirow{2}{*}{$\begin{array}{l}\text { Idade } \\
\text { (anos) }\end{array}$} & \multicolumn{2}{|c|}{$\mathrm{DAP}(\mathrm{cm})$} & \multicolumn{2}{|c|}{ Altura $(\mathrm{m})$} & \multicolumn{2}{|c|}{ Volume total $\left(\mathrm{m}^{3}\right)$} \\
\hline & & $\mathrm{CC}$ & $\mathrm{SC}$ & HT & $\mathrm{HC}$ & $\mathrm{CC}$ & $\mathrm{SC}$ \\
\hline EG & 4 & 15,1 & 13,9 & 22,2 & 18,3 & $0,206565 a$ & $0,175146 a$ \\
\hline EU & 4 & 10,7 & 9,5 & 13,9 & 10,3 & $0,065066 \mathrm{~b}$ & $0,051323 b$ \\
\hline $\mathrm{ECm}$ & 4 & 9,6 & 8,2 & 13,2 & 8,4 & $0,045851 \mathrm{~b}$ & $0,034173 b$ \\
\hline ER & 4 & 11,1 & 9,2 & 13,8 & 9,8 & $0,067319 b$ & $0,050618 b$ \\
\hline $\mathrm{ECl}$ & 4 & 10,7 & 8,7 & 13,5 & 8,5 & $0,054636 \mathrm{~b}$ & $0,038354 b$ \\
\hline EM & 7 & 12,9 & 11,5 & 22,4 & 17,3 & $0,144293 c$ & $0,117119 c$ \\
\hline $\mathrm{EP}$ & 7 & 16,7 & 13,9 & 20,7 & 17,7 & $0,219387 b$ & $0,151294 b$ \\
\hline ET & 7 & 12,2 & 10,8 & 17,9 & 13,6 & $0,104935 c$ & $0,085305 c$ \\
\hline EU & 7 & 14,5 & 13,6 & 20,9 & 17,0 & $0,180042 b$ & $0,146572 b$ \\
\hline HB & 7 & 21,7 & 20,4 & 30,3 & 25,7 & $0,481708 \mathrm{a}$ & $0,418047 \mathrm{a}$ \\
\hline $\mathrm{ECl}$ & 7 & 15,1 & 13,6 & 23,8 & 19,5 & $0,204899 b$ & $0,171497 b$ \\
\hline \multirow{3}{*}{ EP } & 5 & 15,3 & 13,3 & 18,7 & 15,6 & $0,169117 \mathrm{a}$ & $0,124548 \mathrm{a}$ \\
\hline & 6 & 16,3 & 14,1 & 20,7 & 17,7 & $0,216392 a$ & $0,163372 \mathrm{a}$ \\
\hline & 7 & 16,7 & 13,9 & 20,7 & 17,7 & $0,219387 \mathrm{a}$ & $0,151294 a$ \\
\hline Média & & 16,1 & 13,8 & 20,0 & 17,0 & $0,201632 \mathbf{b}$ & $0,146405 b$ \\
\hline \multirow{3}{*}{ EU } & 5 & 11,9 & 10,7 & 15,1 & 11,6 & $0,088025 b$ & $0,069375 b$ \\
\hline & 6 & 10,8 & 9,7 & 15,0 & 11,2 & $0,078284 b$ & $0,062141 b$ \\
\hline & 7 & 14,5 & 13,6 & 20,9 & 17,0 & $0,180042 \mathrm{a}$ & $0,146572 \mathrm{a}$ \\
\hline Média & & 12,4 & 11,3 & 17,0 & 13,3 & $0,115450 \mathbf{c}$ & $0,092696 \mathrm{c}$ \\
\hline \multirow{3}{*}{ HB } & 5 & 15,8 & 14,8 & 25,5 & 20,1 & $0,239256 \mathrm{c}$ & $0,209121 \mathrm{c}$ \\
\hline & 6 & 17,7 & 16,6 & 28,5 & 24,6 & $0,326292 b$ & $0,289058 b$ \\
\hline & 7 & 21,7 & 20,4 & 30,3 & 25,7 & $0,481708 \mathrm{a}$ & $0,418047 \mathrm{a}$ \\
\hline \multirow[t]{4}{*}{ Média } & & 18,4 & 17,3 & 28,1 & 23,4 & $0,349086 \mathbf{a}$ & $0,305408 \mathbf{a}$ \\
\hline & 5 & 14,3 & 12,9 & 19,8 & 15,8 & $0,165466 b$ & $0,134348 b$ \\
\hline & 6 & 14,9 & 13,5 & 21,4 & 17,8 & $0,206989 b$ & $0,171524 b$ \\
\hline & 7 & 17,6 & 16,0 & 24,0 & 20,1 & $0,293713 a$ & $0,238638 \mathrm{a}$ \\
\hline
\end{tabular}

$\mathrm{DAP}=$ diâmetro a $1,3 \mathrm{~m}$ de altura do solo $; \mathrm{CC}$ e $\mathrm{SC}=$ com e sem casca, respectivamente; $\mathrm{HT}=$ altura total; $\mathrm{HC}=$ altura comercial. Valores médios seguidos de mesma letra, em cada coluna, não diferem estatisticamente pelo teste de Scott-Knott a 5\% de probabilidade, para quatro, sete e cinco, seis e sete anos de idade. Diferentes letras minúsculas em negrito, na coluna, indicam diferenças entre as espécies.

estimativa. Para algumas espécies, aos sete anos de idade as estimativas de densidade básica da madeira estão de acordo com Brito et al. (1984).

Pela Tabela 6, verifica-se que, para quatro anos de idade, as espécies mais densas foram o Eucalytpus cloeziana F. Muell. e o E. camaldulensis Dehnh., sendo o E. grandis o de menor densidade básica. Apesar da menor densidade básica média o $E$. grandis apresentou a maior estimativa de massa seca. Observa-se, ainda, que o teste de comparação múltipla para massa seca foi idêntico ao obtido para os volumes, com e sem casca, (Tabela 3), indicando que o volume interfere de maneira decisiva nessa estimativa.

Para a idade de sete anos o E. maculata Hook. e o $E$. tereticornis foram as espécies de maior densidade básica, enquanto que o $E$. urophylla e o híbrido foram os de madeira menos densas. $\mathrm{O}$ híbrido, apesar do baixo valor de densidade básica, foi o de maior estimativa de massa seca, destacandose entre as demais espécies. Esse fato deve-se ao maior incremento volumétrico do híbrido, mesmo comportamento apresentado pelo EG aos quatro anos de idade. O $E$. tereticornis, apesar da elevada densidade básica da madeira, foi a espécie que produziu a menor estimativa de massa seca. 
Tabela 4 - Resumo da análise de variância para a densidade básica, massa seca e de lignina estimada.

\begin{tabular}{|c|c|c|c|c|c|c|}
\hline \multirow[b]{3}{*}{ FV } & \multicolumn{6}{|c|}{ Quatro Anos } \\
\hline & \multicolumn{2}{|c|}{ Densidade Básica $\left(\mathrm{g} / \mathrm{cm}^{3}\right)$} & \multicolumn{2}{|c|}{ Massa Seca (kg) } & \multicolumn{2}{|c|}{ Massa Lignina (kg) } \\
\hline & GL & QM & GL & QM & GL & QM \\
\hline Espécie (E) & 4 & $0,102612 * *$ & 4 & $6620,289181 * *$ & 4 & $510,043158 * *$ \\
\hline Forma $(F)$ & 2 & $0,000602 \mathrm{~ns}$ & 2 & $10,825957 \mathrm{~ns}$ & 2 & $0,851508 \mathrm{~ns}$ \\
\hline$E \times F$ & 8 & $0,000510 \mathrm{~ns}$ & 8 & $9,661492 \mathrm{~ns}$ & 8 & $0,771658 \mathrm{~ns}$ \\
\hline Resíduo & 60 & 0,000916 & 60 & 84,324594 & 60 & 8,179596 \\
\hline \multirow[t]{3}{*}{$\mathrm{CV}(\%)$} & \multicolumn{2}{|c|}{5,74} & \multicolumn{2}{|r|}{27,53} & \multicolumn{2}{|r|}{29,81} \\
\hline & \multicolumn{6}{|c|}{ Sete Anos } \\
\hline & \multicolumn{2}{|c|}{ Densidade Básica $\left(\mathrm{g} / \mathrm{cm}^{3}\right)$} & \multicolumn{2}{|c|}{ Massa Seca (kg) } & \multicolumn{2}{|c|}{ Massa Lignina (kg) } \\
\hline $\mathrm{FV}$ & GL & QM & GL & QM & GL & QM \\
\hline Espécie (E) & 5 & $0,040996 * *$ & 5 & $50603,5685 * *$ & 5 & $3879,516113 * *$ \\
\hline Forma $(\mathrm{F})$ & 2 & $0,002908 \mathrm{~ns}$ & 2 & $202,6632 \mathrm{~ns}$ & 2 & $15,386941 \mathrm{~ns}$ \\
\hline $\mathrm{E} \times \mathrm{F}$ & 10 & $0,000449 \mathrm{~ns}$ & 10 & $93,7017 \mathrm{~ns}$ & 10 & $7,051113 \mathrm{~ns}$ \\
\hline Resíduo & 72 & 0,002120 & 72 & 703,6073 & 72 & 53,819344 \\
\hline $\mathrm{CV}(\%)$ & \multicolumn{2}{|r|}{8,18} & \multicolumn{2}{|r|}{26,72} & \multicolumn{2}{|r|}{27,32} \\
\hline
\end{tabular}

Cinco, Seis e Sete Anos

\begin{tabular}{ccccccc} 
& \multicolumn{2}{c}{ Densidade Básica $\left(\mathrm{g} / \mathrm{cm}^{3}\right)$} & \multicolumn{2}{c}{ Massa Seca $(\mathrm{kg})$} & \multicolumn{2}{c}{ Massa Lignina $(\mathrm{kg})$} \\
\hline FV & GL & QM & GL & QM & GL & QM \\
\hline Espécie (E) & 2 & $0,020196 * *$ & 2 & $130144,5016 * *$ & 2 & $9970,3541 * *$ \\
Idade (I) & 2 & $0,007730 *$ & 2 & $36571,7169 * *$ & 2 & $2786,4576 * *$ \\
Forma (F) & 2 & $0,012517 * *$ & 2 & $637,7387 \mathrm{~ns}$ & 2 & $48,9706 \mathrm{~ns}$ \\
E x I & 4 & $0,009949 * *$ & 4 & $11720,8163 * *$ & 4 & $812,6605 * *$ \\
E x F & 4 & $0,002984 \mathrm{~ns}$ & 4 & $363,8009 \mathrm{~ns}$ & 4 & $27,8651 \mathrm{~ns}$ \\
I x F & 4 & $0,000152 \mathrm{~ns}$ & 4 & $24,5687 \mathrm{~ns}$ & 4 & $1,8176 \mathrm{~ns}$ \\
E x I x F & 8 & $0,000208 \mathrm{~ns}$ & 8 & $12,4797 \mathrm{~ns}$ & 8 & $0,8743 \mathrm{~ns}$ \\
Resíduo & 108 & 0,001638 & 108 & 615,1316 & 108 & 49,4651 \\
\hline CV $(\%)$ & 7,86 & 26,72 & & 27,35 \\
\hline
\end{tabular}

$\mathrm{FV}=$ fonte de variação; $\mathrm{GL}=$ graus de liberdade; $\mathrm{QM}=$ quadrado médio; $\mathrm{CV}=$ coeficiente de variação; $* *$ e $*$ significativo a $1 \mathrm{e}$ $5 \%$ de probabilidade; ns $=$ não significativo Forma $=$ forma de estimativa utilizada . 
Tabela 5 - Resumo da análise de variância para o teor de lignina na madeira.

\begin{tabular}{|c|c|c|c|c|c|c|}
\hline \multirow[b]{2}{*}{ FV } & \multirow[b]{2}{*}{ GL } & \multicolumn{2}{|c|}{ Quadrado Médio } & \multirow[b]{2}{*}{ FV } & \multirow[b]{2}{*}{ GL } & \multirow{2}{*}{$\begin{array}{c}\text { Quadrado Médio } \\
\text { Três Idades }\end{array}$} \\
\hline & & Quatro anos & Sete Anos & & & \\
\hline \multirow[t]{3}{*}{ Espécie } & 4 & $8,584360 * *$ & $26,6462 * *$ & Espécie (E) & 2 & $22,233047 * *$ \\
\hline & & & 0,768553 & Idade (I) & 2 & $4,166487 *$ \\
\hline & & & & Ex I & 4 & $4,143533 * *$ \\
\hline Resíduo & 20 & 0,914268 & & Resíduo & 36 & 0,895570 \\
\hline $\mathrm{CV}(\%)$ & & 3,30 & 3,24 & $\mathrm{CV}(\%)$ & & 3,44 \\
\hline
\end{tabular}

$\mathrm{FV}=$ fonte de variação; $\mathrm{GL}=$ graus de liberdade; $\mathrm{CV}=$ coeficiente de variação; ** $\mathrm{e}^{*}=$ significativo a $1 \mathrm{e} 5 \%$ de probabilidade.

Tabela 6 - Valores médios da densidade básica e massa seca estimada na madeira.

\begin{tabular}{|c|c|c|c|c|c|c|c|c|c|}
\hline \multirow[b]{2}{*}{ Espécie } & \multirow{2}{*}{$\begin{array}{l}\text { Idade } \\
\text { (anos) }\end{array}$} & \multicolumn{3}{|c|}{ Densidade básica $\left(\mathrm{g} / \mathrm{cm}^{3}\right)$} & \multirow{2}{*}{ Média } & \multicolumn{3}{|c|}{ Massa seca estimada (kg) } & \multirow{2}{*}{ Média } \\
\hline & & $\mathrm{DBmP}$ & $\mathrm{DBmA}$ & DBdap & & DBmP & $\mathrm{DBmA}$ & DBdap & \\
\hline EG & 4 & 0,411 & 0,413 & 0,383 & $0,402 d$ & 72,13 & 72,49 & 66,99 & $70,53 a$ \\
\hline EU & 4 & 0,491 & 0,486 & 0,478 & $0,485 c$ & 25,17 & 24,90 & 24,49 & $24,85 b$ \\
\hline $\mathrm{ECm}$ & 4 & 0,587 & 0,590 & 0,570 & $0,582 \mathrm{a}$ & 20,06 & 20,14 & 19,46 & $19,88 \mathrm{~b}$ \\
\hline ER & 4 & 0,562 & 0,554 & 0,567 & $0,561 b$ & 28,42 & 28,01 & 28,69 & $28,37 b$ \\
\hline $\mathrm{ECl}$ & 4 & 0,604 & 0,596 & 0,610 & $0,603 a$ & 23,16 & 22,86 & 23,37 & $23,13 b$ \\
\hline Média & & $0,531 \mathrm{~A}$ & $0,528 \mathrm{~A}$ & $0,521 \mathrm{~A}$ & & $33,79 \mathrm{~A}$ & $33,68 \mathrm{~A}$ & $32,60 \mathrm{~A}$ & \\
\hline EM & 7 & 0,631 & 0,631 & 0,628 & $0,630 \mathrm{a}$ & 73,03 & 73,01 & 72,52 & $72,85 \mathrm{c}$ \\
\hline $\mathrm{EP}$ & 7 & 0,545 & 0,535 & 0,531 & $0,537 \mathrm{c}$ & 82,72 & 80,91 & 80,64 & $81,42 c$ \\
\hline ET & 7 & 0,614 & 0,612 & 0,602 & $0,609 a$ & 52,72 & 52,51 & 51,50 & $52,24 d$ \\
\hline EU & 7 & 0,511 & 0,514 & 0,489 & $0,505 \mathrm{c}$ & 75,50 & 75,95 & 72,47 & $74,64 \mathrm{c}$ \\
\hline $\mathrm{HB}$ & 7 & 0,518 & 0,534 & 0,483 & $0,512 \mathrm{c}$ & 216,23 & 223,03 & 200,99 & $213,42 \mathrm{a}$ \\
\hline $\mathrm{ECl}$ & 7 & 0,589 & 0,585 & 0,575 & $0,583 \mathrm{~b}$ & 102,16 & 101,45 & 99,77 & $101,13 b$ \\
\hline Média & & $0,568 \mathrm{~A}$ & $0,568 \mathrm{~A}$ & $0,551 \mathrm{~A}$ & & $100,39 \mathrm{~A}$ & $101,15 \mathrm{~A}$ & $96,32 \mathrm{~A}$ & \\
\hline \multirow{3}{*}{ EP } & 5 & 0,544 & 0,540 & 0,549 & $0,544 a$ & 67,85 & 67,12 & 68,49 & $67,82 \mathrm{a}$ \\
\hline & 6 & 0,533 & 0,526 & 0,510 & $0,523 a$ & 87,98 & 86,77 & 83,83 & $86,19 a$ \\
\hline & 7 & 0,545 & 0,535 & 0,531 & $0,537 \mathrm{a}$ & 82,72 & 80,91 & 80,64 & $81,42 \mathrm{a}$ \\
\hline Média & & $0,541 \mathrm{~A}$ & $0,534 \mathrm{~A}$ & $0,530 \mathrm{~A}$ & $0,535 \mathbf{a}$ & $79,51 \mathrm{~A}$ & $78,27 \mathrm{~A}$ & $77,65 \mathrm{~A}$ & $78,48 \mathbf{b}$ \\
\hline \multirow{3}{*}{ EU } & 5 & 0,499 & 0,506 & 0,467 & $0,491 b$ & 34,48 & 34,91 & 32,43 & $33,94 b$ \\
\hline & 6 & 0,560 & 0,566 & 0,541 & $0,556 \mathrm{a}$ & 34,67 & 35,08 & 33,53 & $34,43 b$ \\
\hline & 7 & 0,511 & 0,514 & 0,489 & $0,505 b$ & 75,50 & 75,95 & 72,47 & $74,64 \mathrm{a}$ \\
\hline Média & & $0,523 \mathrm{~A}$ & $0,529 \mathrm{~A}$ & $0,499 \mathrm{~A}$ & $0,517 \mathbf{a}$ & $48,22 \mathrm{~A}$ & $48,65 \mathrm{~A}$ & $46,14 \mathrm{~A}$ & $47,67 \mathrm{c}$ \\
\hline \multirow{3}{*}{ HB } & 5 & 0,474 & 0,492 & 0,433 & $0,466 b$ & 99,61 & 103,34 & 91,09 & $98,01 \mathrm{c}$ \\
\hline & 6 & 0,513 & 0,528 & 0,458 & $0,500 \mathrm{a}$ & 149,42 & 153,70 & 133,45 & $145,52 b$ \\
\hline & 7 & 0,518 & 0,534 & 0,483 & $0,512 \mathrm{a}$ & 216,23 & 223,03 & 200,99 & $213,42 \mathrm{a}$ \\
\hline \multirow[t]{4}{*}{ Média } & & $0,502 \mathrm{~A}$ & $0,518 \mathrm{~A}$ & $0,458 \mathrm{~B}$ & $0,493 \mathbf{b}$ & $155,08 \mathrm{~A}$ & $160,02 \mathrm{~A}$ & $141,85 \mathrm{~A}$ & $152,32 \mathbf{a}$ \\
\hline & 5 & & & & $0,500 \mathrm{~b}$ & & & & $66,59 c$ \\
\hline & 6 & & & & $0,526 a$ & & & & $88,71 b$ \\
\hline & 7 & & & & $0,518 \mathrm{a}$ & & & & $123,16 \mathrm{a}$ \\
\hline Média & & $0,522 \mathrm{~A}$ & $0,527 \mathrm{~A}$ & $0,496 \mathrm{~B}$ & & $94,27 \mathrm{~A}$ & $95,65 \mathrm{~A}$ & $88,55 \mathrm{~A}$ & \\
\hline
\end{tabular}

DBmP, DBmA e DBdap = densidade básica média ponderada pelo volume, media aritmética e na altura do DAP, respectivamente. Valores médios seguidos de mesma letra minúscula, na coluna, maiúscula, na linha, não diferem estatisticamente pelo teste de Scott-Knott a 5\% de probabilidade, para quatro, sete e cinco, seis e sete anos de idade. Diferentes letras minúsculas em negrito, na coluna, indicam diferenças entre espécies. 
Para as três diferentes idades, pela Tabela 6, observase que o $E$. pellita $(\mathrm{EP})$ e o $E$. urophylla $(\mathrm{EU})$ apresentaram os maiores valores de densidade básica, sendo estatisticamente diferentes das densidades da madeira do híbrido (HB). Somente o EP não apresentou diferença estatística entre as idades, tanto para a densidade básica como a estimativa de massa seca. O híbrido apresentou tendência evidente de aumento da densidade básica da madeira em função da idade, nas três formas de estimativa utilizadas. A densidade básica da madeira do híbrido foi menor na altura do DAP, sendo diferente da média ponderada pelo volume e média aritmética. $\mathrm{Na}$ estimativa de massa seca, o híbrido foi muito superior e estatisticamente diferente tanto do EP como do EU.

A Tabela 7, apresentam-se os valores médios dos teores de lignina e a massa estimada de lignina na madeira dos materiais avaliados e o teste de comparação múltipla

Tabela 7 - Valores médios do teor de lignina e massa estimada de lignina na madeira.

\begin{tabular}{|c|c|c|c|c|c|c|}
\hline \multirow[b]{2}{*}{ Espécie } & \multirow{2}{*}{$\begin{array}{l}\text { Idade } \\
\text { (anos) }\end{array}$} & \multirow{2}{*}{$\begin{array}{l}\text { LIG } \\
(\%) \\
\end{array}$} & \multicolumn{4}{|c|}{ Massa estimada de lignina $(\mathrm{kg})$} \\
\hline & & & $\mathrm{DBmP}$ & $\mathrm{DBmA}$ & DBdap & Média \\
\hline EG & 4 & $28,04 b$ & 20,32 & 20,43 & 18,87 & $18,87 \mathrm{a}$ \\
\hline EU & 4 & $27,61 b$ & 6,96 & 6,88 & 6,77 & $6,87 b$ \\
\hline $\mathrm{ECm}$ & 4 & $30,19 a$ & 6,05 & 6,07 & 5,87 & $6,00 \mathrm{~b}$ \\
\hline ER & 4 & $30,49 a$ & 8,68 & 8,55 & 8,76 & $8,66 b$ \\
\hline $\mathrm{EC}$ & 4 & $28,37 b$ & 6,58 & 6,49 & 6,64 & $6,57 \mathrm{~b}$ \\
\hline Média & & & $9,72 \mathrm{~A}$ & $9,69 \mathrm{~A}$ & $9,38 \mathrm{~A}$ & \\
\hline EM & 7 & $22,82 \mathrm{c}$ & 16,69 & 16,69 & 16,58 & $16,65 c$ \\
\hline EP & 7 & $29,20 \mathrm{a}$ & 24,10 & 23,57 & 23,46 & $23,71 b$ \\
\hline ET & 7 & $28,92 \mathrm{a}$ & 15,26 & 15,20 & 14,90 & $15,12 \mathrm{c}$ \\
\hline EU & 7 & $27,52 b$ & 20,60 & 20,72 & 19,77 & $20,37 \mathrm{c}$ \\
\hline HB & 7 & $27,34 b$ & 59,24 & 61,10 & 55,06 & $58,47 \mathrm{a}$ \\
\hline $\mathrm{ECl}$ & 7 & $26,45 b$ & 27,05 & 26,86 & 26,41 & $26,77 b$ \\
\hline Média & & & $27,16 \mathrm{~A}$ & $27,36 \mathrm{~A}$ & $26,03 \mathrm{~A}$ & \\
\hline \multirow{3}{*}{ EP } & 5 & $28,06 \mathrm{a}$ & 19,09 & 18,89 & 19,27 & $19,08 \mathrm{~b}$ \\
\hline & 6 & $28,91 \mathrm{a}$ & 25,49 & 25,14 & 24,29 & $24,98 \mathrm{a}$ \\
\hline & 7 & $29,20 \mathrm{a}$ & 24,10 & 23,57 & 23,46 & $23,71 \mathrm{a}$ \\
\hline Média & & $28,72 \mathbf{a}$ & $22,89 \mathrm{~A}$ & $22,53 \mathrm{~A}$ & $22,34 \mathrm{~A}$ & $22,59 \mathbf{b}$ \\
\hline \multirow{3}{*}{ EU } & 5 & $26,44 a$ & 9,14 & 9,26 & 8,60 & $9,00 \mathrm{~b}$ \\
\hline & 6 & $24,90 \mathrm{~b}$ & 8,60 & 8,71 & 8,31 & $8,54 b$ \\
\hline & 7 & $27,52 \mathrm{a}$ & 20,60 & 20,72 & 19,77 & $20,37 \mathrm{a}$ \\
\hline Média & & $26,29 \mathrm{c}$ & $12,78 \mathrm{~A}$ & $12,90 \mathrm{~A}$ & $12,23 \mathrm{~A}$ & $12,64 \mathrm{c}$ \\
\hline \multirow{3}{*}{ HB } & 5 & $28,31 \mathrm{a}$ & 28,26 & 29,32 & 25,83 & $27,80 \mathrm{c}$ \\
\hline & 6 & $27,10 \mathrm{a}$ & 40,56 & 41,69 & 36,14 & $39,46 b$ \\
\hline & 7 & $27,34 \mathrm{a}$ & 59,24 & 61,10 & 55,06 & $58,47 \mathrm{a}$ \\
\hline \multirow[t]{4}{*}{ Média } & & $27,58 \mathbf{b}$ & $42,68 \mathrm{~A}$ & $44,04 \mathrm{~A}$ & $39,01 \mathrm{~A}$ & $41,91 \mathbf{a}$ \\
\hline & 5 & $27,60 b$ & & & & $18,63 \mathrm{c}$ \\
\hline & 6 & $26,97 b$ & & & & $24,33 b$ \\
\hline & 7 & $28,02 \mathrm{a}$ & & & & $34,18 \mathrm{a}$ \\
\hline Média & & & $26,12 \mathrm{~A}$ & $26,49 \mathrm{~A}$ & $24,53 \mathrm{~A}$ & \\
\hline
\end{tabular}

LIG = teor de lignina, DBmP, DBmA e DBdap = densidade básica média ponderada pelo volume, media aritmética e na altura do DAP, respectivamente. Valores médios seguidos de mesma letra minúscula, na coluna, maiúscula, na linha, não diferem estatisticamente pelo teste de Scott-Knott a 5\% de probabilidade, para quatro, sete e cinco, seis e sete anos de idade. Diferentes letras minúsculas em negrito, na coluna, indicam diferenças entre espécies. 
realizado. Para quatro anos de idade observa-se que o EG foi a espécie de destaque na estimativa de massa de lignina na madeira, resultado influenciado pela sua maior estimativa de massa seca. Para sete anos de idade, o ET e o EM foram as espécies que apresentaram as menores estimativas de massa de lignina, enquanto que o $\mathrm{HB}$ foi o destaque nessa estimativa. Para as três idades, observase que o HB foi também o destaque na estimativa de massa de lignina, apresentando tendência evidente de aumento dessa massa com a idade do material. Observa-se também que, para todas as idades avaliadas, o efeito da forma de estimativa utilizada foi não-significativo, resultado igual ao observado na estimativa de massa seca, o que era esperado.

\section{CONCLUSÕES}

Os resultados permitem concluir que:

\section{Volume com e sem casca}

O Eucalyptus grandis (EG), aos quatro anos, e o híbrido (HB), nas demais idades, foram as espécies que se destacaram no incremento volumétrico. Os efeitos de espécie e idade foram significativos para quatro e sete anos de idade. Para cinco, seis e sete anos de idade o efeito da interação espécie x idade também foi significativa, o que era esperado. Somente o E. pellita (EP) não apresentou diferença estatística significativa entre as três idades avaliadas. O HB apresentou tendência de aumento do volume com a idade do material.

\section{Características avaliadas na madeira}

O efeito de espécie foi significativo para a densidade básica, teor de lignina e estimativas de massa seca e de lignina na madeira em todas as idades avaliadas. $\mathrm{O}$ efeito de método foi significativo para a densidade básica somente para o experimento considerando as três idades. O HB foi a espécie que apresentou diferença entre as três formas de estimativa da densidade básica da madeira. O EG foi a espécie que se destacou na produção de massa seca e de lignina, aos quatro anos de idade, enquanto que $\mathrm{HB}$ foi o destaque aos sete e nas três diferentes idades. Essas duas espécies, apesar de não serem as de maior densidade básica e maior teor de lignina na madeira, foram as que promoveram as maiores estimativas de massa.

O incremento volumétrico desempenhou importante papel nas estimativas de massa seca e de lignina na madeira e, portanto, deve ser considerado em todas as utilizações da madeira que depender dessas estimativas.
As formas de estimativa utilizadas apresentaram efeito significativo somente para a densidade básica da madeira. Esse fato ocorreu devido às diferenças de estimativas de densidade básica obtidas para o HB. As estimativas de massa não apresentaram tal comportamento.

\section{REFERÊNCIAS BIBLIOGRÁFICAS}

\section{ASSOCIAÇÃO MINEIRA DE SILVICULTURA. Carvão vegetal. Disponível em: <http://Lww.showsite.com.bra silviminas/html index.asp?Metodo=ExibirLista\&Grupo=2\%20\&SubGrupo=17\%. 2006. Acesso em: 17 ago. 2006.}

BOTREL, M. C. G. Melhoramento genético do Eucalyptus para biomassa florestal e qualidade do carvão vegetal. 2006. 68 p. Dissertação (Mestrado em Engenharia Florestal) - Universidade Federal de Lavras, Lavras, 2006.

BRASIL. Ministério de Minas e Energia. Balanço energético nacional. Brasília, DF, 2005. 188 p.

BRITO, J. O.; BARRICHELO, L. E. G.; COUTO, H. T. Z.; MENDES, C. J.; REZENDE, G. C. Estudo do comportamento de madeira de eucalipto frente ao processo de destilação seca. Revista Brasil Florestal, Brasília, n. 8, p. 5-48, 1984. (Boletim técnico, 8).

DOWNES, G. M.; HUDSON, I. L.; RAYMOND, C. A.; DEAN, G. H.; MICHELL, A. J.; SCHIMLECK, R.; EVANS, R.; MUNERI, A. Sampling plantation eucalypts for wood and fibre properties. [S.1.]: CSIRO, 1997. $126 \mathrm{p}$.

GOLSCHIMID, O. Ultraviolet spectra. In: SARKANEN, K. V.; LUDWIG, C. H. Lignins: occurrence, formation, structure and reations. New York: J. Wiley \& Sons, 1971. p. 241-266.

GOMIDE, J. L.; DEMUNER, B. J. Determinação do teor de lignina em material lenhoso: método Klason modificado. O Papel, São Paulo, v. 47, n. 8, p. 36-38, 1986.

OLIVEIRA, A. D.; LIMA, J. T.; ROSADO, S. C. S.

Estimativa de massa lenhosa em povoamentos de Eucalyptus spp. Ciência e Prática, Lavras, v. 15, n. 4, p. 345-348, 1991. 
TRUGILHO, P. F.; LIMA, J. T.; MENDES, L. M.

Influência da idade nas características físico-químicas da madeira de Eucalyptus saligna. Cerne, Lavras, v. 2, n. 1, p. 94-111, 1996.

VITAL, B. R. Métodos de determinação da densidade da madeira. Viçosa: Sociedade de
Investigações Florestais, 1984. 21 p. (Boletim técnico, 1).

VITAL, B. R.; PEREIRA, A. R.; DELLA-LUCIA, R. M.; ANDRADE, D. C. Efeito da idade da árvore na densidade da madeira de Eucalyptus grandis cultivado na região do cerrado de Minas Gerais. Revista Brasil Florestal, Brasília, n. 8, p. 49-52, 1984. (Boletim técnico, 8). 